\title{
Small-angle neutron scattering study of protein unfolding and refolding
}

\author{
V. K. Aswal, ${ }^{1}$ S. Chodankar, ${ }^{1}$ J. Kohlbrecher, ${ }^{2}$ R. Vavrin, ${ }^{2}$ and A. G. Wagh ${ }^{1}$ \\ ${ }^{1}$ Solid State Physics Division, Bhabha Atomic Research Centre, Mumbai 400 085, India \\ ${ }^{2}$ Laboratory for Neutron Scattering, ETH Zurich and Paul Scherrer Institut, CH-5232 Villigen PSI, Switzerland
}

(Received 19 February 2009; revised manuscript received 2 April 2009; published 29 July 2009)

\begin{abstract}
Small-angle neutron scattering has been used to study protein unfolding and refolding in protein bovine serum albumin (BSA) due to perturbation in its native structure as induced by three different protein denaturating agents: urea, surfactant, and pressure. The BSA protein unfolds for urea concentrations greater than $4 \mathrm{M}$ and is observed to be independent of the protein concentration. The addition of surfactant unfolds the protein by the formation of micellelike aggregates of surfactants along the unfolded polypeptide chains of the protein and depends on the ratio of surfactant to protein concentration. We make use of the dilution method to show the refolding of unfolded proteins in the presence of urea and surfactant. BSA does not show any protein unfolding up to the pressure of $450 \mathrm{MPa}$. The presence of urea and surfactant (for concentrations prior to inducing their own unfolding) has been used to examine pressure-induced unfolding of the protein at lower pressures. The protein unfolds at $200 \mathrm{MPa}$ pressure in the presence of urea; however, no unfolding is observed with surfactant. The protein unfolding is shown to be reversible in all the above denaturating methods.
\end{abstract}

DOI: 10.1103/PhysRevE.80.011924

PACS number(s): 87.14.E-, 87.15.N-, 61.05.fg

\section{INTRODUCTION}

Proteins are responsible for most of the function that occur in living organism and the function of a protein depends absolutely on its three-dimensional folded structure [1-5]. Proteins can undergo unfolding and refolding transitions in the presence of denaturants such as urea, surfactant, and pressure [6-10]. Protein unfolding process involves the disruption of $\mathrm{H}$ bonds, disulphide bonds, salt bridges, and hydrophobic interactions, leading to its successive alteration of quaternary, tertiary, and secondary structures. However, peptide bonds are not broken leaving the primary structure unaltered. Protein unfolding is one of the most widely studied topics in molecular biology due to its widespread application in the industrial and the scientific worlds [11-16]. The unfolding process can be brought about by various means and conditions [17-26]. Each different route of unfolding has its own application and advantage in material processing and basic sciences. Urea is being used for long time to understand the fundamental processes of protein folding and unfolding. On the other hand, the surfactant-induced unfolding is known to play an important role in the pharmaceutical and the cosmetic industries, whereas pressure-induced unfolding is used routinely in food processing industry. Urea is known for its water breaking ability, causing an increase in the solubility of hydrophobic groups, thereby being able to solubilize both the hydrophobic and the hydrophilic patches of the protein macromolecule leading to the unfolding of the whole protein [17-19]. Protein unfolding in the case of amphiphilic molecules such as surfactant is caused due to binding of these molecules to the hydrophobic patches of the protein [20-23]. The effect of applying pressure results in a transfer of solvent to the hydrophobic core of the protein. The weakening of the hydrophobic interactions between the nonpolar side chains due to penetration of solvent results in the pressure-induced unfolding [24-26].

Protein unfolding has been studied using various methods such as viscometry [9], circular dichroism [22], nuclear- magnetic-resonance (NMR), [20] and scattering techniques [23]. These methods probe protein unfolding with different resolutions. For example, viscometry explains the unfolding of proteins based on the increase in viscosity due to the entanglement of unfolded protein [9]. Circular dichroism gives information about the changes in the helical content in the secondary structure, but it does not provide information about the overall changes in the three-dimensional structure of the protein [22]. NMR indicates unfolding from the chemical shift, which is due to the difference in interactions of the folded and the unfolded parts of the protein with the solvent [20]. Scattering techniques correlate the unfolding with the conformational changes in the three-dimensional structure of the protein [23].

Earlier studies have proposed that the addition of urea and the pressure effect unfold a protein into a polypeptide chain, which acquires a random coil conformation [27-31]. In the presence of surfactant, the binding of surfactant on protein results in micellelike aggregates enclosing the hydrophobic patches on the protein backbone. This leads to the acquisition of a necklace-bead structure of the protein-surfactant complex [32-36]. The protein unfolding could be reversible or irreversible depending on its mechanism and the extent of conformation of the unfolded protein [6-10]. Herein, we examine the refolding of the unfolded protein bovine serum albumin (BSA) in the presence of urea, surfactant, and pressure as characterized by the technique of small-angle neutron scattering (SANS). SANS with the possibility to vary the contrast is an ideal technique for studying hydrogenous systems such as protein solution [37]. The experiments are performed on BSA protein, which is one of the commonly used proteins for various studies. BSA functions biologically as a carrier for fatty acids, anions, and other simple amphiphiles in the bloodstream. BSA has a molecular weight of $66.4 \mathrm{kDa}$ and consists of 583 amino acids in a single polypeptide chain. Protein unfolding in the presence of denaturants is studied over a wide concentration range of urea $(0-10 \mathrm{M})$ and surfactant $(0-100 \mathrm{mM})$. The effect of pressure on the protein is observed up to $450 \mathrm{MPa}$. The dilution methods are 
used to examine the refolding of unfolded proteins in these systems.

\section{EXPERIMENT}

BSA protein (Catalog No. 05480), sodium dodecyl sulfate (SDS) surfactant (Catalog No. 71727), and urea were purchased from Fluka. Samples for SANS experiments were prepared by dissolving known amount of BSA and other additives (surfactant or urea) in a buffer solution of $\mathrm{D}_{2} \mathrm{O}$. The use of $\mathrm{D}_{2} \mathrm{O}$ as a solvent instead of $\mathrm{H}_{2} \mathrm{O}$ provides a better contrast for hydrogenous protein in neutron experiments. The interparticle interactions in these systems were minimized by preparing the samples in acetate buffer solution at a $\mathrm{pH}$ of 5.4, which is close to the isoelectric $\mathrm{pH}$ of BSA (4.9), and at a high ionic strength of $0.5 \mathrm{M} \mathrm{NaCl}$. Small-angle neutron scattering experiments were performed at the SANS-I instrument at the Swiss Spallation Neutron Source, SINQ, Paul Scherrer Institut, Switzerland [38]. The mean wavelength of the incident neutron beam was $6 \AA$ with a wavelength resolution of approximately $10 \%$. The scattered neutrons were detected using a two-dimensional $96 \times 96 \mathrm{~cm}^{2}$ detector. The experiments were performed at two sample-to-detector distances of 2 and $8 \mathrm{~m}$, respectively, to cover the data in the wave-vector transfer $Q$ range of $0.006-0.25 \AA^{-1}$. The measured SANS data were corrected and normalized to a crosssectional unit using BERSANS-PC data processing software [39]. Pressure dependence measurements on protein solutions were performed using a $500 \mathrm{MPa}$ high-pressure cell having two parallel thick sapphire windows [40].

\section{SMALL-ANGLE NEUTRON SCATTERING ANALYSIS}

In small-angle neutron scattering, one measures the coherent differential scattering cross section per unit volume $[d \Sigma / d \Omega(Q)]$ as a function of $Q$. For a system of monodispersed interacting protein macromolecules, $d \Sigma / d \Omega(Q)$ can be expressed as [41]

$$
\frac{d \Sigma}{d \Omega}(Q)=N_{p} V_{p}^{2}\left(\rho_{p}-\rho_{s}\right)^{2}\left[\left\langle F(Q)^{2}\right\rangle+\langle F(Q)\rangle^{2}\left(S_{p}(Q)-1\right)\right]+B,
$$

where $N_{p}$ is the protein number density and $V_{p}$ is the volume of the protein macromolecule. $\rho_{p}$ and $\rho_{s}$ are the scattering length densities of the protein and the solvent, respectively. $F(Q)$ is the single-particle form factor and $S_{p}(Q)$ is the interparticle structure factor. $B$ is a constant term that represents the incoherent scattering background, which is mainly due to hydrogen in the sample.

In general, charged colloidal systems such as protein solutions show a correlation peak in the SANS data [42]. The peak arises because of the interparticle structure factor $S_{p}(Q)$ and indicates the presence of significant interaction (electrostatic and/or hard sphere) between the colloids. In the case of a solution with a low protein concentration, having a high salt concentration, and a $\mathrm{pH}$ close to isoelectric point of the protein, $S_{p}(Q)$ can be approximated to unity as the interpar- ticle interactions are minimized, and Eq. (1) for such system becomes

$$
\frac{d \Sigma}{d \Omega}(Q)=N_{p} V_{p}^{2}\left(\rho_{p}-\rho_{s}\right)^{2}\left\langle F(Q)^{2}\right\rangle+B
$$

The single-particle form factor of the protein macromolecules in their native conformation has been calculated by treating them as prolate ellipsoids. For such an ellipsoidal particle, [43]

$$
\begin{aligned}
& \left\langle F(Q)^{2}\right\rangle=\int_{0}^{1}\left[F(Q, \mu)^{2} d \mu\right], \\
& F(Q, \mu)=\frac{3(\sin x-x \cos x)}{x^{3}}, \\
& x=Q\left[a^{2} \mu^{2}+b^{2}\left(1-\mu^{2}\right)\right]^{1 / 2},
\end{aligned}
$$

where $a$ and $b$ are, respectively, the semimajor and the semiminor axes of the ellipsoidal protein macromolecules and $\mu$ is the cosine of the angle between the directions of $a$ and the wave-vector transfer $Q$.

The unfolding of protein in the presence of urea or on applying pressure is believed to be the opening of the globular protein structure into a random coil Gaussian conformation of the unfolded polypeptide chain. In this case, scattering cross section is given as [44]

$$
\frac{d \Sigma}{d \Omega}(Q)=I_{0}\left[Q^{2} R_{g}^{2}-1+\exp \left(-Q^{2} R_{g}^{2}\right)\right] /\left(Q R_{g}\right)^{4},
$$

where $R_{g}$ is the radius of gyration of the unfolded protein polypeptide chain.

The protein-surfactant complex has been treated using the necklace model that assumes micellelike clusters of surfactant randomly distributed along the unfolded polypeptide chain. The cross section for such a system can be written as [45]

$$
\frac{d \Sigma_{s}}{d \Omega}(Q)=\frac{N_{1}^{2}}{N_{p} N}\left(b_{m}-V_{m} \rho_{s}\right)^{2} P_{m}(Q) S_{f}(Q)+B,
$$

where $N_{1}$ and $N_{p}$ are the number densities of the total surfactant and the protein molecules in the solution, respectively. $V_{m}$ is the volume of the surfactant and $N$ is the number of micelles attached to a polypeptide chain. $b_{m}$ represents the scattering length of the surfactant molecule. The aggregation number of the micellelike clusters in the complex is obtained by $n=N_{1} /\left(N_{p} N\right) . P_{m}(Q)$ denotes the normalized intraparticle structure factor of a single micellelike cluster, which for a spherical micelles of radius $R$ is given by

$$
P_{m}(Q)=\left[\frac{3(\sin Q R-Q R \cos Q R)}{(Q R)^{3}}\right]^{2}
$$

$S_{f}(Q)$ has been calculated using fractal structure of the complex. In this case, $S_{f}(Q)$ is given as [46] 

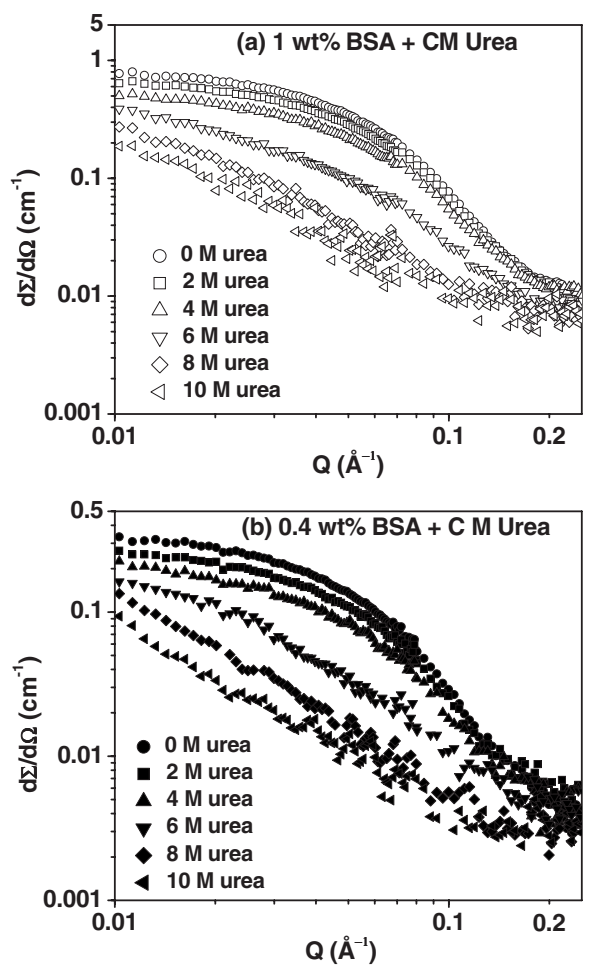

FIG. 1. SANS data for (a) 1 and (b) 0.4 wt $\%$ BSA in the presence of varying urea concentration.

$$
\begin{aligned}
S_{f}(Q)= & 1+\frac{1}{\left(Q R_{p}\right)^{D}} \frac{D \Gamma(D-1)}{\left[1+(Q \xi)^{-2}\right]^{[(D-1) / 2]}} \\
& \times \sin \left[(D-1) \tan ^{-1}(Q \xi)\right],
\end{aligned}
$$

where $D$ is the fractal dimension of the gel and $\xi$ is the correlation length that is a measure of the extent of the complex. Throughout the data analysis, corrections were made for instrumental smearing. The parameters in the analysis were optimized by means of nonlinear least-square fitting program and the errors (standard deviations) on the parameters were calculated by the standard method [47].

\section{RESULTS AND DISCUSSION}

SANS data for two concentrations (1 and $0.4 \mathrm{wt} \%$ ) of BSA protein in the presence of varying concentration of urea are shown in Fig. 1. The effect of addition of urea shows similar behavior for both the protein concentrations. SANS data show a decrease in scattering cross section with an increasing urea concentration. It is observed that, up to $4 \mathrm{M}$ concentration of urea, there is a continuous decrease in the scattering cross section; however the functionality of the scattering pattern does not change. The decrease in scattering cross section can be explained in terms of a decrease in contrast $\left(\rho_{p}-\rho_{s}\right)^{2}$ as the scattering length density of deuterated solvent $\left(\rho_{s}\right)$ decreases in addition of hydrogenous urea to protein solution. There is a change in the functionality of the scattering profile beyond $4 \mathrm{M}$ urea and it is interpreted in terms of the unfolding of the protein. It is believed that the solvation of hydrophobic portions of the protein at high urea concentrations leads to the unfolding of a protein [17-19].

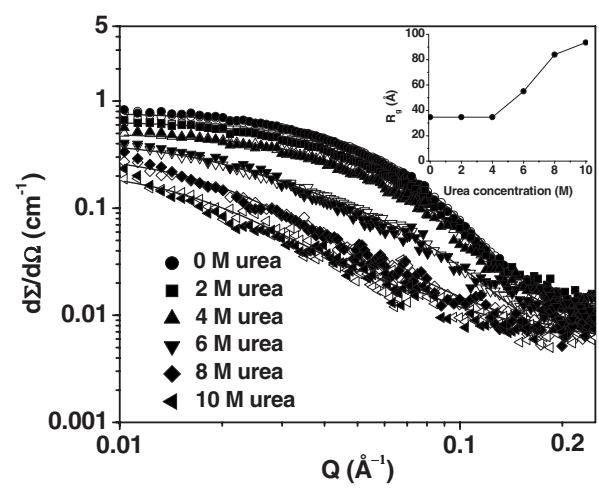

FIG. 2. Scaling of the SANS data of 1 and $0.4 \mathrm{wt} \%$ BSA in the presence of urea. The data of $0.4 \mathrm{wt} \% \mathrm{BSA}$ (filled symbols) are multiplied by a factor of 2.5 (concentration factor) to scale with the data of 1 wt \% BSA (open symbols). Inset shows the variation in radius of gyration $\left(R_{g}\right)$ of protein as a function of urea concentration.

Figure 2 shows the scaling of the data of two BSA concentrations (Fig. 1) by the concentration factor. The overlapping of the two data sets suggests that the effect of urea is independent of the BSA concentration in the present concentration range of interest. SANS data for the native structure of a protein have been fitted using Eq. (2). It is found that the protein macromolecules are prolate ellipsoidal in shape with semimajor and semiminor axes $a=71.0$ and $b=c=22.2 \AA$, respectively, which are similar to the values reported earlier [23]. Recently, a more detailed model based on the crystal structure of protein has been developed which could provide a better idea about the quality of the scattering data as well as the prolate ellipsoidal model [48]. The unfolded protein is fitted as random Gaussian coil using Eq. (6). It is found that the radius of gyration $\left(R_{g}\right)$ of the unfolded protein increases with increasing urea concentration. The value of $R_{g}$ increases from 55.0 to $93.5 \AA$ as the urea concentration is increased from 6 to $10 \mathrm{M}$ (Table I). It may be mentioned that, while random Gaussian coil model of unfolded protein is not completely realistic, we believe that a wormlike chain with excluded volume effect would be more appropriate [49]. This model has three unknowns, namely, the contour length, the Kuhn length, and the cross-section radius to fit the data.

TABLE I. Fitted parameters of SANS analysis for BSA protein in the presence of varying urea concentration. The protein has a prolate ellipsoidal shape up to $4 \mathrm{M}$ urea and beyond this concentration it unfolds into a random coil conformation. The parameters are independent of the BSA concentration.

\begin{tabular}{lccc}
\hline \hline $\begin{array}{l}\text { Urea } \\
(\mathrm{M})\end{array}$ & $\begin{array}{c}\text { Semiminor axis } \\
b=c(\AA)\end{array}$ & $\begin{array}{c}\text { Semimajor axis } \\
a(\AA)\end{array}$ & $\begin{array}{c}\text { Radius of gyration } \\
R_{g}(\AA)\end{array}$ \\
\hline 0 & $22.2 \pm 0.8$ & $71.0 \pm 5.1$ & \\
2 & $22.2 \pm 0.8$ & $71.0 \pm 5.1$ & \\
4 & $22.2 \pm 0.8$ & $71.0 \pm 5.1$ & \\
6 & & & $55.0 \pm 2.9$ \\
8 & & & $84.0 \pm 4.1$ \\
10 & & & $93.5 \pm 6.4$ \\
\hline \hline
\end{tabular}




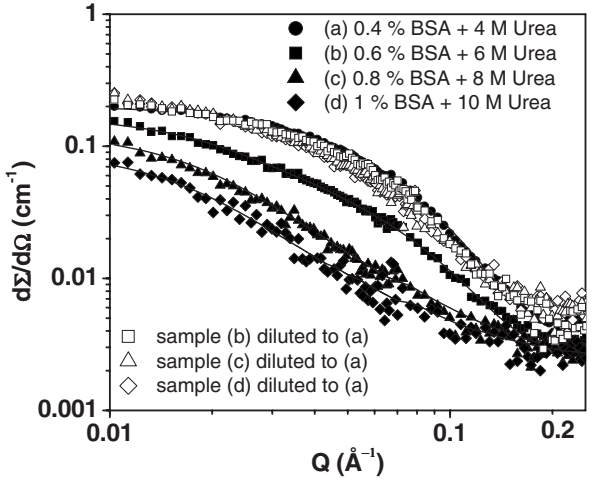

FIG. 3. SANS data of $1 \mathrm{wt} \% \mathrm{BSA}+10 \mathrm{M}$ urea, $0.8 \mathrm{wt} \%$ $\mathrm{BSA}+8 \mathrm{M}$ urea, and $0.6 \mathrm{wt} \% \mathrm{BSA}+6 \mathrm{M}$ urea, and these samples are diluted to $0.4 \mathrm{wt} \%+4 \mathrm{M}$ urea. All the data are scaled to 1 wt $\%$ BSA concentration.

However, we have not used this model due to limitations to fit these three parameters as the SANS data are taken over the limited $Q$ range and scattering cross section has quite low values.

The refolding of unfolded protein in the presence of urea has been examined using dilution method and the data are shown in Fig. 3. In this method, the urea concentration in the protein solution is reduced through the dilution of the sample. The results can be directly compared as the protein unfolding is independent of the protein concentration. Figure 3 shows the scaled SANS data of 1 wt $\% \mathrm{BSA}+10 \mathrm{M}$ urea, 0.8 wt $\% \mathrm{BSA}+8 \mathrm{M}$ Urea, and 0.6 wt $\% \mathrm{BSA}+6 \mathrm{M}$ urea to $1 \mathrm{wt} \%$ protein data and all these systems are diluted to 0.4 wt $\% \mathrm{BSA}+4 \mathrm{M}$ urea by factors of $2.5,2$, and 1.5 , respectively. The comparison of scattering profiles of $1 \mathrm{wt} \%$ $\mathrm{BSA}+10 \mathrm{M}$ urea, 0.8 wt $\% \mathrm{BSA}+8 \mathrm{M}$ urea, and 0.6 wt $\%$ $B S A+6 \mathrm{M}$ urea systems suggests the higher amount of unfolding at higher urea concentrations. However, very similar data are obtained when the protein solution with different urea concentrations (unfolded proteins) are diluted to one urea concentration at which the protein is refolded. This is a clear indication of the unfolded protein in $1 \mathrm{wt} \%$ BSA with 6-10 M urea concentration range getting back to the folded structure on reducing the urea concentration by the dilution method. The structural details of unfolded and refolded proteins in these systems are given in Table II. The small differ-
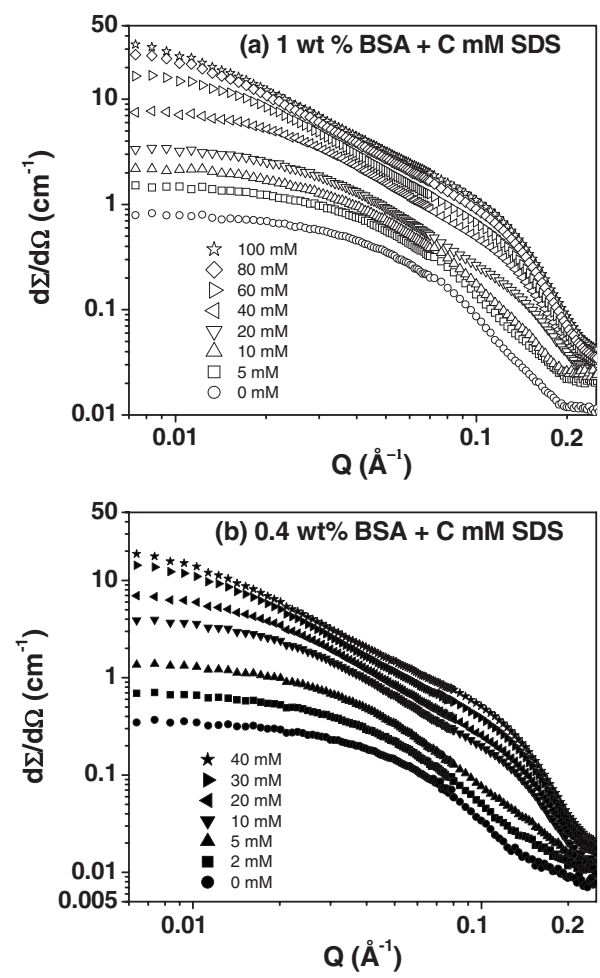

FIG. 4. SANS data for (a) 1 and (b) 0.4 wt $\%$ BSA in the presence of varying SDS concentration.

ence between refolded structures of protein from different amounts of unfolding of the protein on dilution may be due to that protein unfolding is not completely reversible and the extent of refolding depends on the unfolding state of the protein.

Figure 4 shows the SANS data for BSA ( 1 and 0.4 wt \%) in the presence of varying SDS concentration. The effect of addition of surfactant unlike urea depends on the BSA concentration, and lower surfactant concentration is required for low protein concentration to obtain similar scattering profiles. SANS data of both the BSA concentrations show an increase in scattering cross section with an increasing surfactant concentration. Based on the features of the scattering profiles, the data can be grouped in two different sets as the surfactant concentration is increased. The first data set corresponds to proteins at low surfactant concentrations, where

TABLE II. Fitted parameters of SANS analysis of unfolded and refolded proteins as a function of urea concentration. The protein has a prolate ellipsoidal shape in the folded state and a random coil conformation in unfolded state.

\begin{tabular}{lcccc}
\hline \hline System & $\begin{array}{c}\text { Conformational } \\
\text { state }\end{array}$ & $\begin{array}{c}\text { Semiminor axis } \\
b=c(\AA)\end{array}$ & $\begin{array}{c}\text { Semimajor axis } \\
a(\AA)\end{array}$ & $\begin{array}{c}\text { Radius of gyration } \\
R_{g}(\AA)\end{array}$ \\
\hline (a) $1 \mathrm{wt} \%$ BSA+4 M Urea & Folded & $22.2 \pm 0.8$ & $71.0 \pm 5.1$ & \\
(b) $1 \mathrm{wt} \% \mathrm{BSA}+10 \mathrm{M}$ Urea & Unfolded & & & $93.5 \pm 6.4$ \\
(c) $0.8 \mathrm{wt} \%$ BSA+8 M Urea & Unfolded & & & $84.0 \pm 4.1$ \\
(d) $0.6 \mathrm{wt} \%$ BSA+6 M Urea & Unfolded & & $88.0 \pm 6.3$ & \\
Sample (b) diluted to sample (a) & Refolded & $22.2 \pm 0.8$ & \\
Sample (c) diluted to sample (a) & Refolded & $22.2 \pm 0.8$ & $82.0 \pm 5.8$ & \\
Sample (d) diluted to sample (a) & Refolded & $22.2 \pm 0.8$ & $75.0 \pm 5.5$ & \\
\hline \hline
\end{tabular}




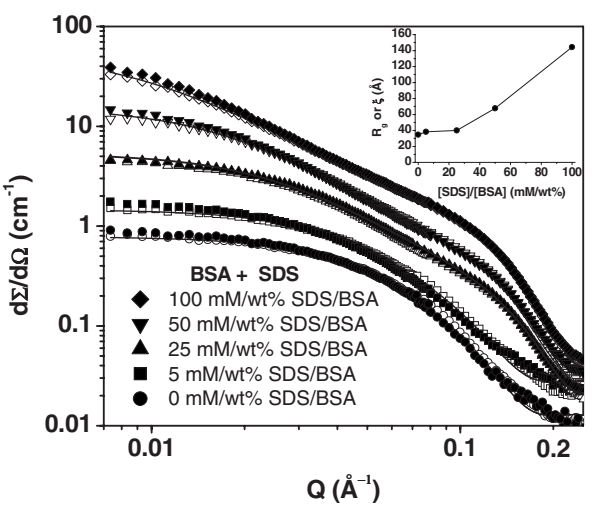

FIG. 5. Scaling of the SANS data of 1 and 0.4 wt \% BSA in the presence of SDS. The data are shown for the systems for which the ratio of surfactant to protein concentration is same. The data of 0.4 wt $\%$ BSA (filled symbols) are multiplied by a factor of 2.5 (concentration factor) to scale with the data of $1 \mathrm{wt} \%$ BSA (open symbols). Inset shows the variation in radius of gyration $\left(R_{g}\right)$ or correlation length $(\xi)$ of protein as a function of ratio of SDS to BSA concentration.

the scattering data show a similar behavior to that of pure protein solution. In this data set, the overall scattering cross section increases with an increase in surfactant concentration. It can be explained in terms of Eq. (2) if the individual surfactant molecules bind to protein and the volume of the scattering particle increases. The features of the scattering data in the second data set at higher surfactant concentrations are very different to those of the first data set. One of the interesting features is the linearity of the scattering profiles on log-log scale in the intermediate- $Q$ range with a $Q$ range of linearity increasing with the surfactant concentration. This is an indication of the formation of fractal structure by the protein-surfactant complex. The buildup of scattering cross section in the higher cutoff of the linearity of scattering data suggests the formation of surfactant aggregates, and the lower cutoff corresponds to the overall size of the protein-

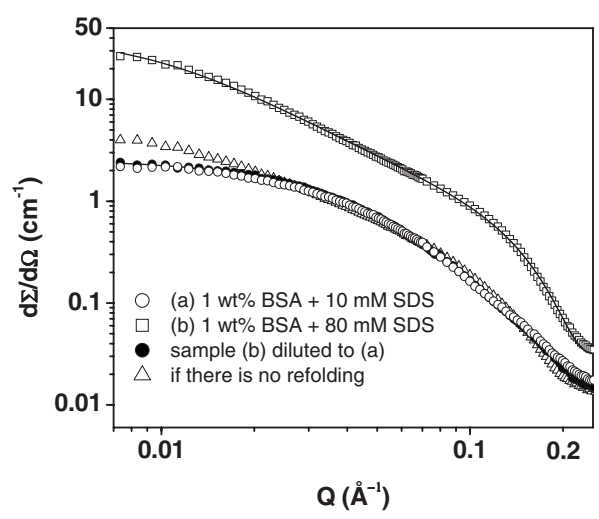

FIG. 6. SANS data for 1 wt $\%$ BSA $+80 \mathrm{mM}$ SDS and this system is diluted to $1 \mathrm{wt} \% \mathrm{BSA}+10 \mathrm{mM}$ SDS. The data of the diluted sample are compared with that of the directly prepared sample by the mixing of two components. The calculated data are also shown if there is no refolding on dilution.

surfactant complex. It is observed that the position of high- $Q$ cutoff remains almost the same while the position of low- $Q$ cutoff shifts to smaller $Q$ values with increasing surfactant concentration. It is interesting to note that when the data of adding surfactant concentration are scaled with respect to protein concentration (Fig. 5), the similar scattering profiles are obtained from Figs. 4(a) and 4(b) for those systems which have the same surfactant to protein ratios. In other words, these results show that the scattering profile in protein-surfactant system depends on the ratio of surfactant to protein concentration. The calculated structural parameters in these systems are given in Table III.

At low surfactant concentrations, Table III shows changes in the dimensions of the protein on an increase in binding of surfactant molecules as a function of surfactant concentration. The semiminor axis remains almost the same while the semimajor axis increases with increasing surfactant concentration. Similar results of the elongation of the BSA protein have also been observed with cationic surfactant azobenzene

TABLE III. Fitted parameters of SANS analysis of protein-surfactant complexes. Data are fitted for prolate ellipsoidal shape of the protein macromolecule for folded structure and by a fractal structure of micellelike clusters randomly distributed along the unfolded protein chain for unfolded structure.

(a) Folded structure

\begin{tabular}{lcc}
\hline $\begin{array}{l}{[\mathrm{SDS}] /[\mathrm{BSA}]} \\
(\mathrm{mM} / \mathrm{wt} \%)\end{array}$ & $\begin{array}{c}\text { Semiminor axis } \\
b=c(\AA)\end{array}$ & $\begin{array}{c}\text { Semimajor axis } \\
a(\AA)\end{array}$ \\
\hline 0 & $22.2 \pm 0.8$ & $71.0 \pm 5.1$ \\
5 & $22.2 \pm 0.8$ & $80.0 \pm 6.1$
\end{tabular}

(b) Unfolded structure

\begin{tabular}{|c|c|c|c|c|c|}
\hline $\begin{array}{l}{[\mathrm{SDS}] /[\mathrm{BSA}]} \\
(\mathrm{mM} / \mathrm{wt} \%)\end{array}$ & $\begin{array}{c}\text { Fractal } \\
\text { dimension } \\
D\end{array}$ & $\begin{array}{c}\text { Correlation } \\
\text { length } \\
\xi(\AA)\end{array}$ & $\begin{array}{c}\text { Micelle } \\
\text { radius } \\
R(\AA)\end{array}$ & $\begin{array}{c}\text { Number } \\
\text { of micelles } \\
N\end{array}$ & $\begin{array}{c}\text { Aggregation } \\
\text { number } \\
n\end{array}$ \\
\hline 25 & $2.23 \pm 0.15$ & $40.0 \pm 1.9$ & $18.0 \pm 0.6$ & 2 & 51 \\
\hline 50 & $1.95 \pm 0.10$ & $67.8 \pm 4.9$ & $18.0 \pm 0.6$ & 6 & 45 \\
\hline 100 & $1.71 \pm 0.04$ & $144.3 \pm 7.5$ & $18.0 \pm 0.6$ & 13 & 42 \\
\hline
\end{tabular}




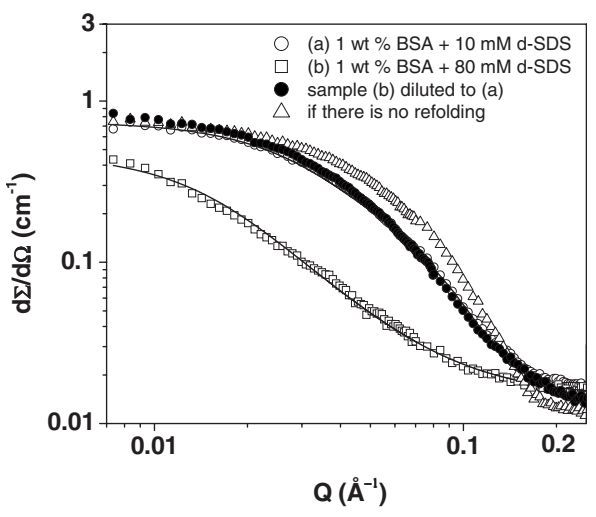

FIG. 7. SANS data with contrast-matched surfactant for 1 wt $\%$ $\mathrm{BSA}+80 \mathrm{mM} d$-SDS and this system diluted to 1 wt $\%$ BSA $+10 \mathrm{mM} d$-SDS. The data of the diluted sample are compared with that of the directly prepared sample by the mixing of two components. The data are also shown if there is no refolding on dilution.

trimethyl ammonium bromide. It is believed that the six protein subdomains forming BSA remain intact but separates from each other, leading to an elongation of the protein on addition of surfactant [50]. The fractal structure of the protein-surfactant complex at higher surfactant concentrations is modeled on the basis of the necklace model that considers micellelike clusters of the surfactant formed along the unfolded polypeptide chain of the protein. It is found that the fractal dimension decreases and the overall size of the complex increases on increasing surfactant concentration. The size of micellelike clusters $(R)$ does not change, while the number of such micellelike clusters $(N)$ in proteinsurfactant complex increases with the surfactant concentration. The values of aggregation numbers are much smaller than that one would have found, about 70 in pure surfactant solution for the similar size of micelles [51]. This indicates the participation of the hydrophobic portions of the unfolded protein chain in the micellar formation [20-23]. The participation of the unfolded protein in the formation of micellelike clusters is enhanced with the increase in unfolding, and this results in a decreasing aggregation number of micellelike clusters. Also, all the surfactant molecules probably participate in the micellelike clusters to avoid the exposure of hydrophobic portions of the protein on its unfolding with an increase in surfactant concentration.

We again use the dilution method to examine the refolding of surfactant-induced protein unfolding. The fact that protein unfolding depends on the ratio of protein to surfactant concentration suggests that the dilution of both compo-

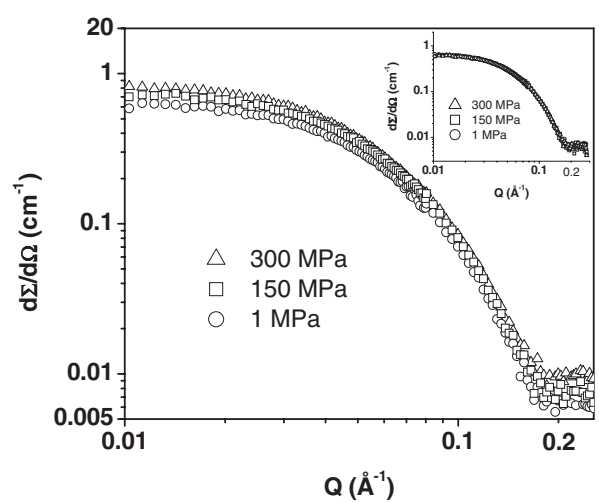

FIG. 8. SANS data for 1 wt $\%$ BSA as a function of pressure. Inset shows the scaling of SANS data.

nents as in the case of urea cannot be used to examine the refolding. Therefore, native protein solution (pure BSA) has been used to dilute the surfactant-induced unfolded protein. Figure 6 shows the SANS data of unfolded protein structure of 1 wt $\%$ BSA+80 mM SDS diluted by 1 wt $\%$ BSA to 1 wt $\%$ BSA $+10 \mathrm{mM}$ SDS for which the protein is expected to be in the folded structure. The scattering from diluted sample is found to be exactly similar to that of directly prepared sample of $1 \mathrm{wt} \%$ BSA+10 mM SDS suggesting that unfolded protein refolds as the surfactant concentration is decreased through dilution. Figure 6 also shows the theoretically generated scattering data if there was no refolding of protein in $1 \mathrm{wt} \% \mathrm{BSA}+80 \mathrm{mM} \mathrm{SDS}$ on dilution, which is found to be quite different to that experimentally measured. It is calculated by the weighted scattering sum of the unfolded (1 wt \% BSA $+80 \mathrm{mM}$ SDS) and the folded (1 wt \% BSA) proteins in the diluted sample. These results thus suggest that surfactant molecules redistribute themselves depending on the protein concentration and hence the unfolded structure can be refolded back on decreasing the surfactant concentration. Figure 7 shows the similar data to Fig. 6 using contrast-matched (deuterated) surfactant. In this case only the protein conformation is visible and the data are fitted to the Gaussian random coil for the unfolded protein. These data also show the refolding of the unfolded protein on dilution.

Figure 8 shows the SANS data for pressure effect on 1 wt $\%$ protein solution. It is found that there is an increase in scattering cross section on applying the pressure. However, the functionality of the scattering profile remains the same (see inset of the figure). The increase in scattering cross section can be understood in terms of the increase in number

TABLE IV. Fitted parameters of SANS analysis of 1 wt $\%$ BSA protein on applying pressure.

\begin{tabular}{|c|c|c|c|c|c|}
\hline \multirow[b]{2}{*}{$\begin{array}{l}\text { Pressure } \\
(\mathrm{MPa})\end{array}$} & \multicolumn{2}{|c|}{ Protein structure } & \multicolumn{3}{|c|}{ Factor of increase in scattering cross section } \\
\hline & $\begin{array}{l}\text { Semiminor axis } \\
\quad b=c(\AA)\end{array}$ & $\begin{array}{c}\text { Semimajor axis } \\
a(\AA)\end{array}$ & Total & $\begin{array}{l}\text { Number density } \\
\text { contribution }\end{array}$ & $\begin{array}{l}\text { Contrast factor } \\
\text { contribution }\end{array}$ \\
\hline 1 & $22.2 \pm 0.8$ & $71.0 \pm 5.1$ & 1 & 1 & 1 \\
\hline 150 & $22.2 \pm 0.8$ & $71.0 \pm 5.1$ & 1.15 & 1.08 & 1.07 \\
\hline 300 & $22.2 \pm 0.8$ & $71.0 \pm 5.1$ & 1.24 & 1.16 & 1.07 \\
\hline
\end{tabular}




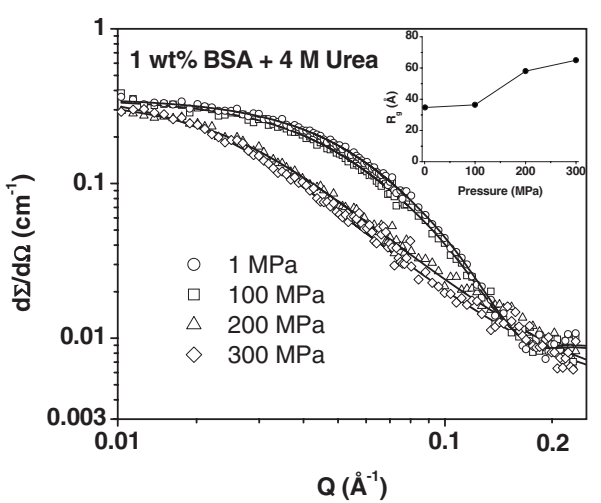

FIG. 9. SANS data for $1 \mathrm{wt} \% \mathrm{BSA}+4 \mathrm{M}$ urea as a function of pressure. Inset shows the variation in radius of gyration $\left(R_{g}\right)$ of protein as a function of pressure.

density of the protein macromolecules and the scattering length density of the solvent (i.e., contrast factor) as the system gets compressed on applying pressure. The individual calculated contributions of these two terms are given in Table IV. The number density of the protein macromolecules increases with the pressure, and the contrast factor does not change perhaps due to the increase in the hydration of the protein. It is also found that there is no any change in the structure of BSA protein upon pressure, and these results are consistent with our earlier dynamic light scattering (DLS) data that this protein does not show any unfolding even up to pressure of $450 \mathrm{MPa}$ [31]. Pressure-induced unfolding is believed to be due to water penetration inside the core of the protein that decreases the hydrophobic interactions among nonpolar groups of the protein molecule [24-26]. It has been found for small globular proteins such as staphylococcal nuclease (SN) [30] that pressure beyond $200 \mathrm{MPa}$ can unfold these proteins. We do not observe any protein unfolding by applying pressure up to $450 \mathrm{MPa}$; it may be perhaps due to the fact that BSA $(66.4 \mathrm{KDa})$ is a much larger protein than SN $(16.8 \mathrm{KDa})$ for which pressure-induced unfolding has been observed in this pressure range. Larger size of the protein means higher hydrophobic interactions between the nonpolar groups inside the protein [52] and therefore larger pressure is needed to weaken these interactions. The size may not only matter, it could also be due to differences in the dominance of the different interactions within the protein structure [6].

Since the BSA protein does not unfold on applying pressure up to $450 \mathrm{MPa}$, we have used pressure effect in the

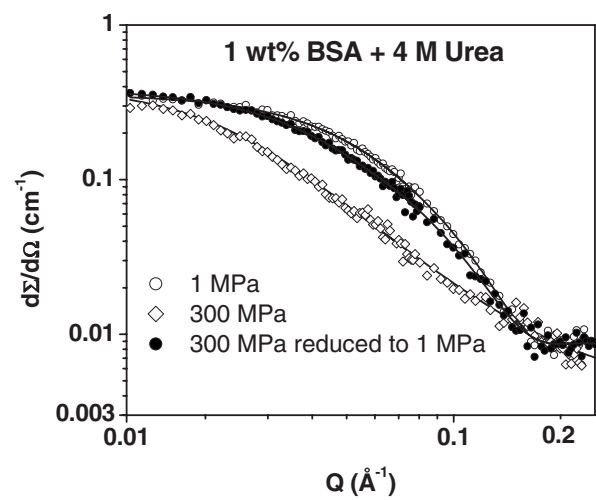

FIG. 10. SANS data for 1 wt $\% \mathrm{BSA}+4 \mathrm{M}$ urea at $300 \mathrm{MPa}$ and when this pressure is released to $1 \mathrm{MPa}$.

presence of urea and surfactant prior to unfolding by their own. SANS experiments are performed on 1 wt $\%$ BSA $+4 \mathrm{M}$ urea and $1 \mathrm{wt} \% \mathrm{BSA}+10 \mathrm{mM}$ SDS. Figure 9 shows the SANS data of pressure effect on $1 \mathrm{wt} \%+4 \mathrm{M}$ urea. It is seen that there is a significant change in the scattering profile at $200 \mathrm{MPa}$, which is an indication of the protein unfolding similar to that of addition of higher urea concentration $(>4 \mathrm{M})$. The maximum pressure is applied up to $300 \mathrm{MPa}$ and no drastic changes in protein unfolding are seen as compared to that for $200 \mathrm{MPa}$. The refolding of unfolded protein is examined by releasing the pressure from 300 to $1 \mathrm{MPa}$. The scattering pattern suggests the refolding of the unfolded protein on removing the pressure. The fitted parameters of the folded and the unfolded protein in these systems are given in Table $\mathrm{V}$. The globular protein unfolds to random coil conformation at $200 \mathrm{MPa}$ and only shows a small increase on further increasing the pressure to $300 \mathrm{MPa}$. On releasing the pressure, protein is refolded back to globular size (Fig. 10). The refolded size is found to be little larger than that at its native state.

Figure 11 shows the pressure effect on 1 wt $\%$ BSA $+10 \mathrm{mM} d$-SDS, where the surfactant is contrast matched with the solvent to see directly the conformation of protein. There is no significant change in the scattering profiles suggesting that the presence of surfactant unlike urea shows only partial unfolding to protein up to the pressure of 350 $\mathrm{MPa}$. The protein maintains its globular structure in $1 \mathrm{wt} \%$ $\mathrm{BSA}+10 \mathrm{mM} d$-SDS on applying pressure (Table VI). However, there is an increase in the size of the proteinsurfactant complex. We do not observe unfolding of protein

TABLE V. Fitted parameters of SANS analysis of pressure effect on $1 \mathrm{wt} \% \mathrm{BSA}+4 \mathrm{M}$ Urea. The protein has a prolate ellipsoidal shape in the folded or refolded state and a random coil conformation in unfolded state.

\begin{tabular}{lcccc}
\hline \hline $\begin{array}{l}\text { Pressure } \\
(\mathrm{MPa})\end{array}$ & $\begin{array}{c}\text { Conformational } \\
\text { state }\end{array}$ & $\begin{array}{c}\text { Semiminor axis } \\
b=c(\AA)\end{array}$ & $\begin{array}{c}\text { Semimajor axis } \\
a(\AA)\end{array}$ & $\begin{array}{c}\text { Radius of gyration } \\
R_{g}(\AA)\end{array}$ \\
\hline 1 & Folded & $22.2 \pm 0.8$ & $71.0 \pm 5.1$ & \\
100 & Folded & $22.2 \pm 0.8$ & $75.0 \pm 5.5$ & \\
200 & Unfolded & & & $58.0 \pm 3.0$ \\
300 & Unfolded & & & $65.0 \pm 4.0$ \\
1 & Refolded & $22.2 \pm 0.8$ & $80.5 \pm 6.0$ & \\
\hline \hline
\end{tabular}




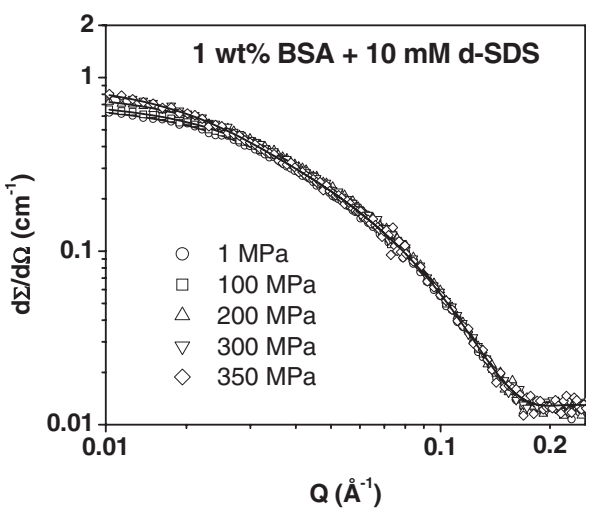

FIG. 11. SANS data for 1 wt $\% \mathrm{BSA}+10 \mathrm{mM} d$-SDS as a function of pressure.

to random coil with surfactant; it could be due to the fact that the pressure effect prevents the formation of micellelike clusters with the protein as the surfactant micellization is known to disfavor with increasing pressure [53]. As a result unlike urea the effect of addition of surfactant does not add to that of the pressure on protein unfolding. To the best of our knowledge, we have not found any literature on BSA where the activity of protein has been examined on refolding. However, we believe that the refolding of protein in the present studies is acquired to achieve a native structure. In the case of any misfolding [7], it would have resulted in aggregation, which was not observed in any of these systems.

\section{CONCLUSIONS}

The unfolding and/or refolding of BSA protein have been studied using SANS as functions of different variables such as urea, surfactant, and pressure. The addition of urea leads to the unfolding of the protein only at concentrations higher than $4 \mathrm{M}$ and is observed to be independent of the protein
TABLE VI. Fitted parameters of SANS analysis of pressure effect on 1 wt $\%$ BSA $+10 \mathrm{mM} d$-SDS. The protein maintains its prolate ellipsoidal shape up to pressure of $350 \mathrm{MPa}$.

\begin{tabular}{lcc}
\hline \hline $\begin{array}{l}\text { Pressure } \\
(\mathrm{MPa})\end{array}$ & $\begin{array}{c}\text { Semiminor axis } \\
b=c(\AA)\end{array}$ & $\begin{array}{c}\text { Semimajor axis } \\
a(\AA)\end{array}$ \\
\hline 1 & $22.2 \pm 0.8$ & $88.0 \pm 5.5$ \\
100 & $22.2 \pm 0.8$ & $88.0 \pm 5.5$ \\
200 & $22.2 \pm 0.8$ & $94.0 \pm 6.0$ \\
300 & $22.2 \pm 0.8$ & $100.0 \pm 6.5$ \\
350 & $22.2 \pm 0.8$ & $105.0 \pm 7.0$ \\
\hline \hline
\end{tabular}

concentration. For urea concentrations above $4 \mathrm{M}$, the protein unfolds and acquires a random coil Gaussian conformation, whose radius of gyration increases with an increasing urea concentration. The unfolding of protein in the presence of surfactant depends on the ratio of surfactant to protein concentration and is found to be caused by the formation of micellelike clusters along the unfolded protein polypeptide chain. There is no protein unfolding observed for pure BSA on the application of pressure up to $450 \mathrm{MPa}$. However, the presence of urea $(4 \mathrm{M})$ shows the pressure-induced unfolding of BSA protein to random coil conformation at $200 \mathrm{MPa}$ pressure. No unfolding is observed with surfactant (1 wt \% BSA+10 mM SDS) system even up to pressure of $350 \mathrm{MPa}$. Dilution methods are used to examine the reversibility of the protein unfolding and it has been found that the unfolded protein in the presence of all the above denaturants refolds back to the folded structure.

\section{ACKNOWLEDGMENT}

This work was based on SANS experiments performed at the Swiss spallation neutron source SINQ, Paul Scherrer Institut, Villigen, Switzerland.
[1] C. B. Anfinsen, Biochem. J. 128, 737 (1972).

[2] T. E. Creighton, Protein Folding (W. H. Freeman \& Company, New York, 1992).

[3] T. E. Creighton, Protein Function: A Practical Approach (Oxford University Press, Oxford, 1997).

[4] A. Fersht, Structure and Mechanism in Protein Science (W. H. Freeman, New York, 1999).

[5] M. A. Dwyer, L. L. Looger, and H. W. Hellinga, Science 304, 1967 (2004).

[6] C. Tanford, Adv. Protein Chem. 24, 1 (1970).

[7] C. M. Dobson, Nature (London) 426, 884 (2003).

[8] D. Paschek and A. E. Garcia, Phys. Rev. Lett. 93, 238105 (2004).

[9] R. S. Tu and V. Breedveld, Phys. Rev. E 72, 041914 (2005).

[10] R. Day and V. Daggett, J. Mol. Biol. 366, 677 (2007).

[11] A. H. Clark, G. M. Kavanagh, and S. B. Ross-Murphy, Food Hydrocolloids 15, 383 (2001).

[12] T. Hagiwara, H. Kumagai, and K. Nakamura, Food Hydrocol- loids 12, 29 (1998).

[13] R. S. Meyer, K. L. Cooper, D. Knorr, and H. L. M. Lelieveld, Food Technol. 54, 67 (2000).

[14] E. D. Goddard and K. P. Ananthapadmanabhan, Interactions of Surfactants with Polymers and Proteins (CRC Press, London, 1993).

[15] C. Sun, J. Yang, X. Wu, X. Huang, F. Wang, and S. Liu, Biophys. J. 88, 3518 (2005).

[16] C. C. Chang, Y. C. Su, M. S. Cheng, and L. S. Kan, Phys. Rev. E 66, 021903 (2002).

[17] C. Tanford, J. Am. Chem. Soc. 86, 2050 (1964).

[18] A. Wallqvist, D. G. Covell, and D. Thirumalai, J. Am. Chem. Soc. 120, 427 (1998).

[19] B. J. Bennion and V. Daggett, Proc. Natl. Acad. Sci. U.S.A. 100, 5142 (2003).

[20] N. J. Turro, X.-G. Lei, K. P. Ananthapadmanabhan, and M. Aronson, Langmuir 11, 2525 (1995).

[21] A. Valstar, M. Almgren, W. Brown, and M. Vasilescu, Lang- 
muir 16, 922 (2000)

[22] S. Deep and J. C. Ahluwalia, Phys. Chem. Chem. Phys. 3, 4583 (2001).

[23] S. Chodankar, V. K. Aswal, J. Kohlbrecher, R. Vavrin, and A. G. Wagh, J. Phys.: Condens. Matter 19, 326102 (2007).

[24] G. Panick, R. Malessa, and R. Winter, Biochemistry 38, 6512 (1999).

[25] R. Ravindra and R. Winter, ChemPhysChem 4, 359 (2003).

[26] Y. Harano and M. Kinoshita, J. Phys.: Condens. Matter 18, L107 (2006).

[27] A. Vassilikou-Dova, S. Grigorakakis, P. Varotsos, A. Kapetanaki, and D. Koutsikos, J. Phys. Chem. 100, 1914 (1996).

[28] L. Chen, K. O. Hodgson, and S. Doniach, J. Mol. Biol. 261, 658 (1996).

[29] P. Garcia, L. Serrano, D. Durand, M. Rico, and M. Bruix, Protein Sci. 10, 1100 (2001).

[30] A. Paliwal, D. Asthagiri, D. P. Bossev, and M. E. Paulaitis, Biophys. J. 87, 3479 (2004).

[31] S. Chodankar, V. K. Aswal, J. Kohlbrecher, R. Vavrin, and A. G. Wagh, Phys. Rev. E 77, 031901 (2008).

[32] X. H. Guo, N. M. Zhao, S. H. Chen, and J. Teixeira, Biopolymers 29, 335 (1990).

[33] M. Vasilescu, D. Angelescu, M. Almgren, and A. Valstar, Langmuir 15, 2635 (1999).

[34] A. Stenstam, G. Montalvo, I. Grill, and M. Gradzielski, J. Phys. Chem. B 107, 12331 (2003).

[35] E. L. Gelamo, R. Itri, A. Alonso, J. V. da Silva, and M. Tabak, J. Colloid Interface Sci. 277, 471 (2004).

[36] S. Chodankar, V. K. Aswal, P. A. Hassan, and A. G. Wagh, Physica B 398, 112 (2007).
[37] D. Svergun and M. H. J. Koch, Rep. Prog. Phys. 66, 1735 (2003).

[38] J. Kohlbrecher and W. Wagner, J. Appl. Crystallogr. 33, 804 (2000).

[39] U. Keiderling, Appl. Phys. A: Mater. Sci. Process. 74, S1455 (2002).

[40] J. Kohlbrecher, A. Bollhalder, R. Vavrin, G. Meier, and J. Buitenhuis, Rev. Sci. Instrum. 78, 125101 (2007).

[41] J. B. Hayter and J. Penfold, Colloid Polym. Sci. 261, 1022 (1983).

[42] S. Chodankar and V. K. Aswal, Phys. Rev. E 72, 041931 (2005).

[43] J. S. Pedersen, Adv. Colloid Interface Sci. 70, 171 (1997).

[44] P. Debye, J. Phys. Colloid Chem. 51, 18 (1947).

[45] S. H. Chen and J. Teixeira, Phys. Rev. Lett. 57, 2583 (1986).

[46] J. Teixeira, J. Appl. Crystallogr. 21, 781 (1988).

[47] P. R. Bevington, Data Reduction and Error Analysis for Physical Science (McGraw-Hill, New York, 1969).

[48] C. D. Putnam, M. Hammel, G. L. Hura, and J. A. Tainer, Q. Rev. Biophys. 40, 191 (2007).

[49] J. S. Pedersen and P. Schurtenberger, Macromolecules 29, 7602 (1996).

[50] C. Ted Lee, Jr., K. A. Smith, and A. T. Hatton, Biochemistry 44, 524 (2005).

[51] V. K. Aswal and P. S. Goyal, Phys. Rev. E 67, 051401 (2003).

[52] C. Tanford, The Hydrophobic Effect: Formation of Micelles and Biological Membranes (Wiley, New York, 1980).

[53] D. P. Bossev, S. R. Kline, J. N. Israelachvili, and M. E. Paulaitis, Langmuir 17, 7728 (2001). 